\title{
Measuring Gastrointestinal Electrical Activity With Extracellular Electrodes: Author's Reply
}

TO THE EDITOR: In our recent paper ${ }^{1}$ we found that 2 points of intracellular microelectrode recording were insufficient to describe significant disruptions in gastric motor patterns that were caused by inhibition of acetylcholinesterase. Thus, we employed video imaging and analyzed data by image processing routines developed by Dr Hennig. ${ }^{1}$ The resulting motility maps described the aberrant patterns of gastric contractions when tissue cholinesterases were inhibited in much more detail that could be achieved using electrical recording. In describing our rationale for the technology chosen, we made reference to the unsuitability of extracellular electrical recording as a means to measure changes in slow waves, because movement artifacts dominate these measurements. ${ }^{2}$ In a previous study we found that when movements are blocked, under conditions in which slow waves are unaffected, the signals recorded with extracellular metal electrodes and many have attributed to electrophysiological events (slow waves), are inhibited. ${ }^{2}$ Thus the signals monitored by extracellular electrodes are largely artifacts of movement and do not represent authentic slow wave activity. In light of these observations, which have been reproduced in mouse, dog, monkey and human gastric muscles when movement is suppressed, it is important for studies utilizing extracellular electrical recording to be accompanied by rigorous tests insuring that the electrical signals claimed to be slow waves persist after movement is stabilized.

O'Grady et $\mathrm{al}^{3}$ have invested a great effort in writing letters to editors when we have commented in several papers on the unsuitability of extracellular recording for electrophysiological measurements in GI muscles. We feel compelled to comment on the artifacts of extracellular electrical recording, because this technique has been used and continues to be used for research studies and for patient evaluations, yet adequate validation of the technique has not been provided. Extracellular array electrodes might be selected as a means to record integrated signals from slow waves, smooth muscle electrical activity and muscle contractions in the absence of other recording techniques, but the steps required to "deconvolute" these signals would be extremely complex. Modeling such signals as descriptions of gastric electrophysiology seems folly. In our view a better use of time than writing letters would be to perform rigorous and convincing control experiments that demonstrate unequivocally the degree to which movements contaminate extracellular signals. Neglecting such control experiments to exclude the possibility of movement artifacts will continue to cloud their results, and it was this skepticism about the validity of extracellular recordings and our own empirical tests that caused us to avoid this technique in our recent study. ${ }^{1}$

O'Grady et $\mathrm{al}^{3}$ claim they have performed adequate controls for movement ${ }^{4}$ but the tests they performed stopped well short of providing convincing evidence that their recordings are not the result of movement artifacts. As we have detailed previously, it may not be feasible to stabilize movements of muscular tissues in vivo to the extent required to remove any suspicion of movement artifacts from extracellular recordings in GI muscles. ${ }^{5}$ We have directly measured movement artifacts in smooth muscle sheets that are similar to the waveforms of the signals O'Grady et $\mathrm{al}^{3}$ consider as "ideal" electrical recording in response to movements of less than $50 \mu \mathrm{m}$. Respiratory movements, circulation of blood and muscle contractions uncontrolled by dihydropyridines are all possible sources of such tiny movements in the in vivo tests they performed. ${ }^{4}$ We have suggested that the best approach to make clear association between extracellular recording and authentic slow waves is rigorous stabilization of movements in vitro, as described in the addendum of our previous paper. ${ }^{2}$ If one is determined to monitor contraction by just looking at the tissue, then relatively high power objectives would be needed to allow observations of the tiny movements that cause significant signals (ie, 
several 10 seconds of $\mu V_{s}$ ) to be picked up by extracellular electrodes. We fully realize the time, effort and resources that O'Grady et $\mathrm{al}^{3}$ have invested in extracellular recording and modeling the data derived from these measurements, and we sincerely hope new ideas might be developed to alleviate movement artifacts or amplify the field potentials caused by slow waves to a level where they can be captured by extracellular recording. But as things stand, this technique has not been validated as a measurement of the field potentials resulting from the transmembrane currents occurring during electrical slow waves, and as such, its authenticity in describing electrophysiological events in visceral smooth muscles will continue to be questioned. If, as claimed, extracellular recording is the "gold standard" for interpretation of slow wave frequency and patterns in gastrointestinal muscles, then how about providing a rigorous assay of the gold?

Kenton M Sanders and Grant Hennig Department of Physiology and Cell Biology, University of Nevada School of Medicine, Reno, NV, USA
1. Worth AA, Forrest AS, Peri LE, Ward SM, Hennig GW, Sanders KM. Regulation of gastric electrical and mechanical activity by cholinesterases in mice. J Neurogastroenterol Motil 2015;21:200-216.

2. Bayguinov O, Hennig GW, Sanders KM. Movement based artifacts may contaminate extracellular electrical recordings from GI muscles. Neurogastroenterol Motil 2011;23:1029-1042, e498.

3. O'Grady G, Angeli TR, Du P, Cheng LK. Measuring gastrointestinal electrical activity with extracellular electrodes. J Neurogastroenterol Motil 2015;21:623-624.

4. Angeli TR, Du P, Paskaranandavadivel N, et al. The bioelectrical basis and validity of gastrointestinal extracellular slow wave recordings. J Physiol 2013;591(Pt 18):4567-4579.

5. Sanders KM, Ward SM, Koh SD. Interstitial Cells: Regulators of Smooth Muscle Function. Physiol Rev 2014;94:859-907.

\section{Conflicts of interest: None.}

